\title{
Uso racional de la energía eléctrica y eficiencia energética aplicado al edificio $4 \mathrm{~A}$ en la UNAH
}

Dennis A. Rivera ${ }^{1}$

Naun R. Olguín ${ }^{2}$

Roger F. Nieto ${ }^{2}$

\section{RESUMEN}

Actualmente el país vive una crisis energética debido a que la producción de energía eléctrica en su mayoría es térmica, aproximadamente en un 70\%. Esta situación de crisis surge, primero por los altos precios de los combustibles, ya que la generación de energía eléctrica en nuestro país depende del Bunker; segundo porque no se han implementado proyectos de generación de energía en base a recursos renovables con la suficiente capacidad y celeridad como para desplazar a la generación térmica; y tercero por el consumo excesivo de energía eléctrica. Ante estas situaciones, nos vemos obligados todos los hondureños a implementar una eficiencia energética en nuestros hogares, en la industria y en los edificios públicos y privados. La UNAH no queda exenta de esta crisis energética y se ve obligada a ejercer un plan de ahorro de energía en cada uno de sus edificios. En esta investigación presentamos alternativas viables de ahorro de energía en el sistema de iluminación del edificio 4-A, y lo más importante de este proyecto es que no ocupa una inversión monetaria, lo cual hace que sea factible casi en un 100\%, y consiste en la modificación de la conexión del temporizador y una adecuada programación de los tiempos en el temporizador. Asimismo se encontró que el transformador de alimentación del edificio está sobrecargado y debe ser reemplazado en carácter de urgencia.

palabras clave: Energía eléctrica, crisis energética, consumo de energía, ahorro de energía.

\section{ABSTRACT}

The country currently lives an energy crisis due to the fact that $70 \%$ of the generated

\footnotetext{
${ }^{1}$ Catedrático Seminario de Investigación IE-900

${ }^{2}$ Estudiantes de Ingeniería Eléctrica
} 
electricity comes from fuel powered generators. The crisis surges because of the high costs of fuel on which the country's electrical generation rely on, and because clean energy resources capable to displace the fuel generated electricity have not been implemented and finally by a high consumption of electricity. In the presence of this situation, all Hondurans are compelled to implement energy efficiency on our homes, industries and in public and private buildings. The UNAH is not exempt from this energy crisis and it has to implement a viable energy saving plan on the illumination system of the 4-A building, and the most important part of this program is that it does not require a monetary investment which makes it feasible in almost a $100 \%$. The plan consists in the modification of the electric timer and a adequate setting on the times on the timer. It was also found that the electric transformer of the building is overcharged and requires an urgent replacement.

Key Words: Electrical energy, energy crisis, energy consumption, energy saving. 


\section{INTRODUCCIÓN}

El término eficiencia energética se refiere a la implementación de cambios, mejoras, modificaciones en los procesos, actividades u operaciones, que lleven consigo la intención de propiciar un ahorro energético o una mayor eficiencia energética. En nuestro caso es obtener una mayor eficiencia en el sistema de iluminación del edificio 4-A y de esta forma reducir el pago del gasto eléctrico. Por consiguiente, los métodos que permitan incrementar la eficiencia energética sirven para reducir los costos y las emisiones de contaminación y de esta forma beneficiar a la economía nacional mediante el aumento de la actividad comercial.

\section{Formas de aumentar la eficiencia energética.}

Existen varios campos de aplicación dentro de los cuales podemos mencionar los siguientes:

- Reduciendo las horas de funcionamiento de las lámparas.

- Programando el temporizador en las horas que en realidad se necesita ocupar las lámparas.

- A través de rótulos publicitarios colocándolos en el edificio para fomentar una cultura de ahorro energético, tanto en los estudiantes, los maestros, así como personal que labora en la universidad.

\section{DETALLES GENERALES EN EL EDIFICIO 4-A}

\section{Equipo de medición instalado en el edificio 4-A}

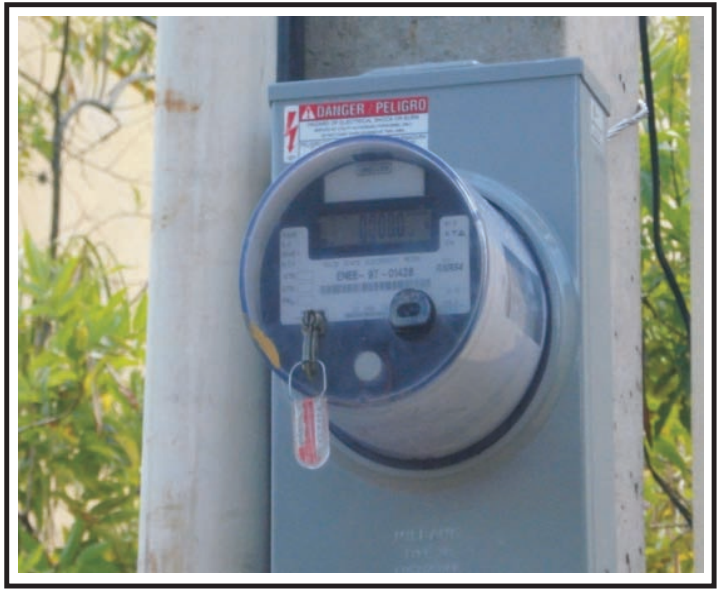

Fig. 1 Analizador de red eléctrica instalado en el edificio 4-A. 


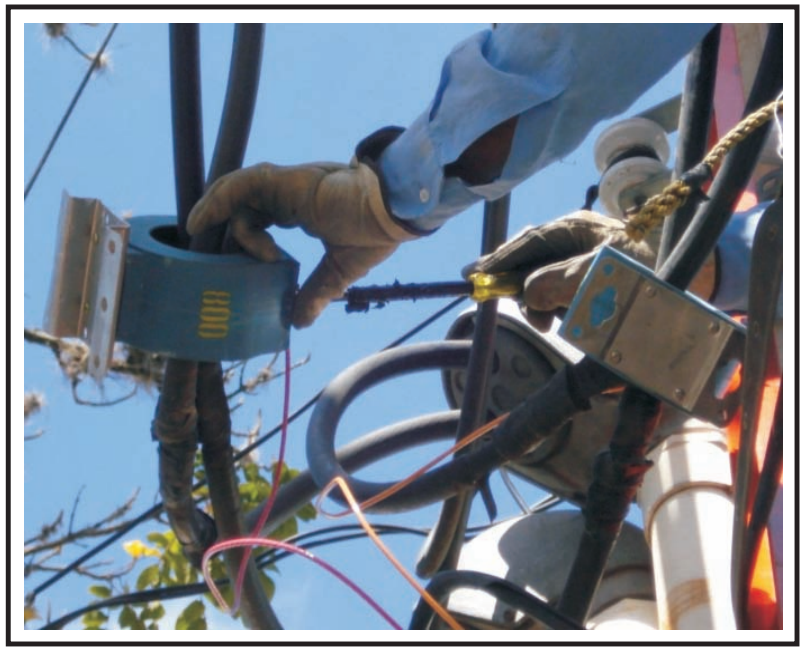

Fig. 2 Transformador de corriente instalado en el edificio 4-A.

\section{Equipo de alimentación en el edificio 4-A.}

Este edificio está alimentado por un transformador de 100 KVA conectado en delta; el cableado del secundario es de $650 \mathrm{Kcmil}$ ( 2 por fase), tiene un main principal de 600 A y sus breakers de distribución son de 200 A. En la Fig. 3 se muestra el transformador que alimenta el edificio 4-A:

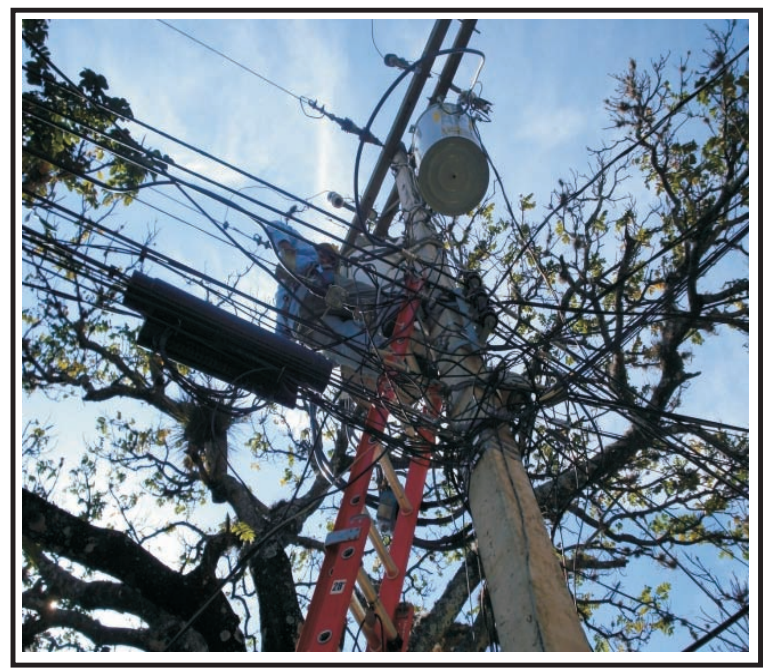

Fig. 3 Transformador de alimentación en el edificio 4-A. 


\section{Equipo de control instalado en el edificio 4-A}

Este edificio está controlado por un temporizador que controla los tiempos de encendido de las lámparas de las aulas de clases y de los pasillos. Está programado para activar las lámparas de 9:00 am hasta las 10:30 am y de 3:30 pm hasta las 10:30 pm, y representa un consumo innecesario durante la jornada diurna; realizamos un estudio de ahorro energético durante este lapso de tiempo. En laFig. 4 se muestra el temporizador que controla el sistema de encendido de las lámparas del edificio 4-A:

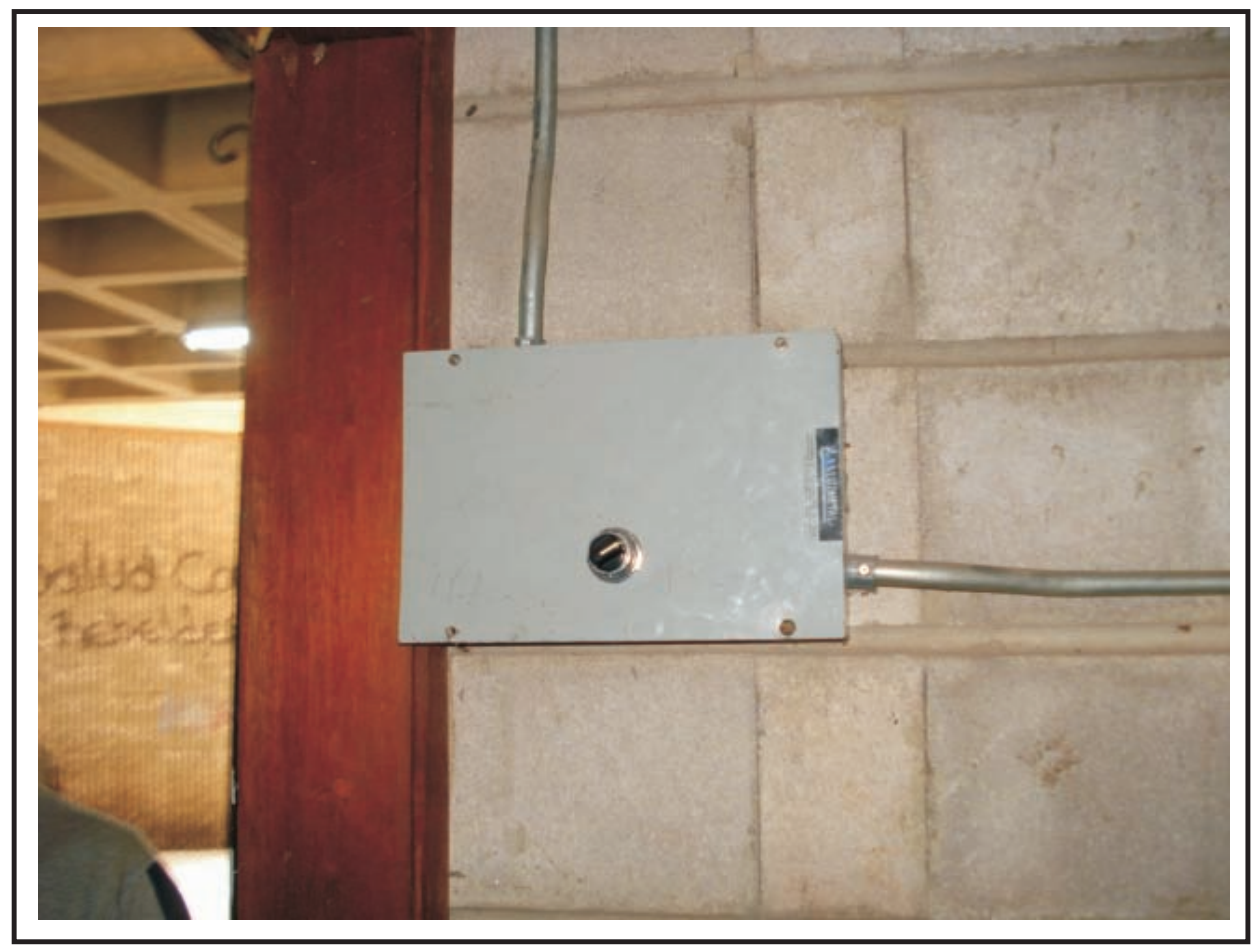

Fig. 4 Temporizador instalado en el edificio 4-A

\section{CONSUMO DE ENERGÍA EN EL EDIFICIO4-A}

En la Tabla I se presenta un resumen del consumo de energía que se registró en el edificio 4-Aen el III periodo del 2007: 
Tabla I. Resumen del consumo energía en el edificio 4-A durante el tercer periodo del 2007 [2]

\begin{tabular}{|l|c|c|}
\hline \multicolumn{1}{|c|}{ Tipo de uso } & $\begin{array}{c}\text { Energía } \\
\text { (KWH/día) }\end{array}$ & $\begin{array}{c}\text { Energía } \\
\text { (KWH/mes) }\end{array}$ \\
\hline Aire acondicionado & 193 & 4237.2 \\
\hline Equipo de cómputo & 755.55 & 16622.1 \\
\hline Iluminación & 842.88 & 18543.36 \\
\hline Ventiladores & 31.5 & 693 \\
\hline Fotocopiadoras & 594 & 13068 \\
\hline Equipo de cocina + pequeños aparatos & 130.4 & 2868.8 \\
\hline TOTAL & 2547 & 56032.46 \\
\hline
\end{tabular}

En la Fig. 5 se muestra el porcentaje de consumo de energía en el edificio 4-A:

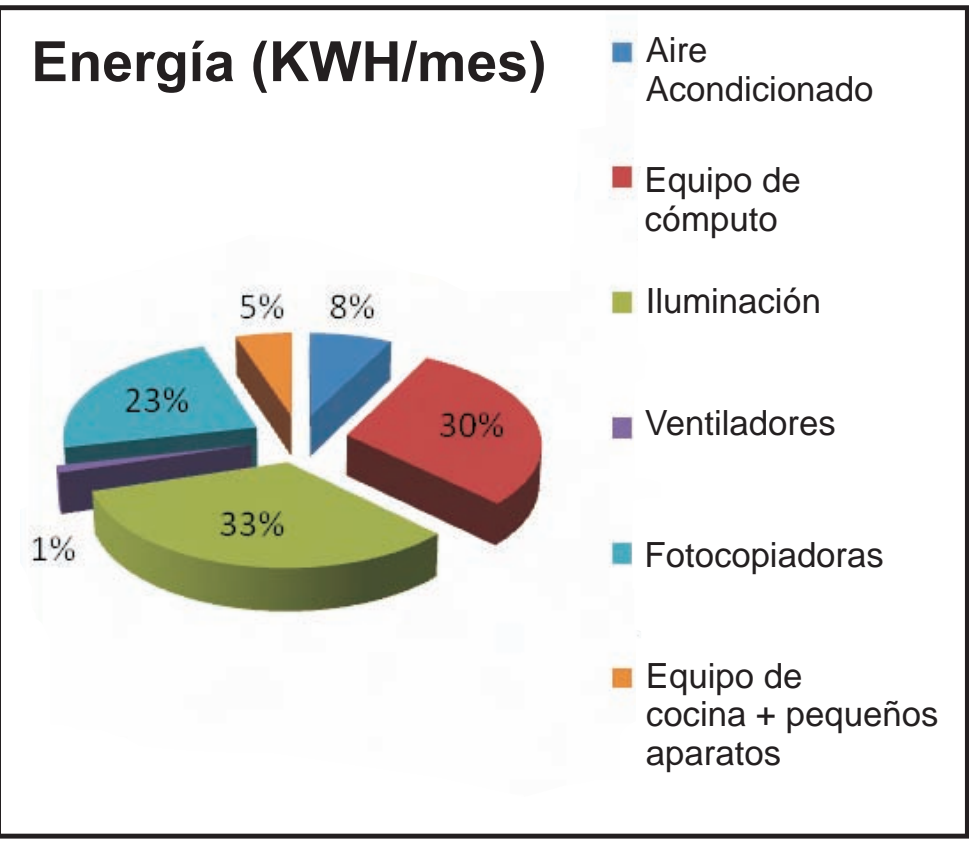

Fig. 5 Porcentaje de consumo de energia en el edificio 4-A. 


\section{Consumo actual en el edificio 4-A}

Actualmente nuestra investigación de eficiencia energética referido al edificio 4-A está basada a partir de mediciones reales proporcionadas por el personal que labora en la ENEE. El equipo de medición logró registrar valores de potencia activa, potencia reactiva, factor de potencia, voltaje y corriente. En la Tabla II se muestra el consumo actual que existe en el sistema de iluminación del edificio 4-A:

Tabla II. Consumo actual que existe en el sistema de iluminación del edificio 4-A

\begin{tabular}{|l|c|c|c|c|}
\hline & Potencia (KW) & $\begin{array}{c}\text { Horas de } \\
\text { funcionamiento }\end{array}$ & $\begin{array}{c}\text { Consumo } \\
\text { diario (KWH) }\end{array}$ & $\begin{array}{c}\text { Consumo } \\
\text { mensual (KWH) }\end{array}$ \\
\hline Baños & 2.88 & 24 & 69.12 & 2073.6 \\
\hline Pasillos & 4.68 & 8.5 & 39.78 & 835.38 \\
\hline Aulas & 9.4 & 8.5 & 79.9 & 1677.9 \\
\hline Oficinas & 16 & 10 & 160 & 3360 \\
\hline $\begin{array}{l}\text { Iluminación } \\
\text { externa }\end{array}$ & 5 & 10 & 50 & 1500 \\
\hline TOTAL & 37.96 & & 398.8 & 9446.88 \\
\hline
\end{tabular}

El consumo actual de las restantes cargas (aire acondicionado, equipo de cómputo, ventiladores, fotocopiadoras, equipo de cocina y otros aparatos) del edificio 4-A es de $30,711.144 \mathrm{KWH}$.

En la Fig. 6 se muestra el porcentaje de consumo de energía actual en el edificio 4-A: 


\section{ENERGÍA (KWH/mes)}

\section{lluminación}

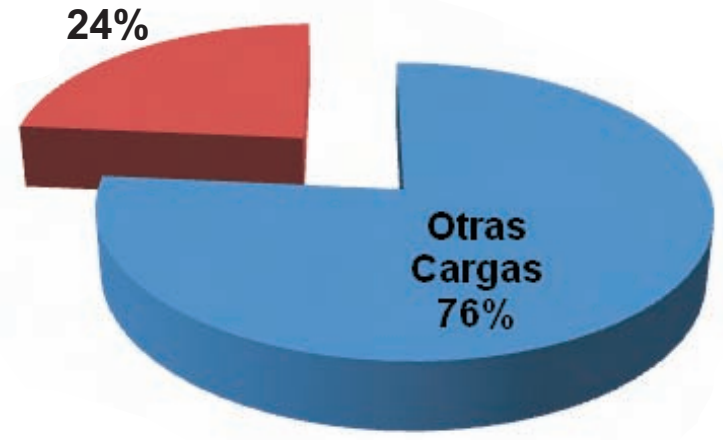

Fig. 6 Porcentaje de consumo de energia actual en el edificio 4-A

A partir de la Fig. 6 podemos afirmar que el porcentaje de iluminación registrado actualmente, ha tenido un decrecimiento en comparación con el porcentaje obtenido por el grupo de eficiencia energética que llevó el seminario de investigación en el III periodo del 2007 [2]. Este decrecimiento se debe a que ha crecido la carga en el sistema de aire acondicionado, y también ha aumentado considerablemente la carga en el sistema de computo. Este aumento en la carga del sistema de computo, se debe a que se han instalado más computadoras en los laboratorios de matematicas, lenguas extranjeras y psicologia.

\section{GRÁFICOS GENERALES DEL EDIFICIO 4-A}

\section{Gráficos de energía.}

A partir de las mediciones que se obtuvieron en el analizador de red, logramos obtener la Fig. 7, en donde se muestra la energía consumida cada 15 minutos en el edificio 4-A durante una semana, a partir del 16 de abril hasta el 23 de abril del 2008 y tambien se muestra la energía consumida en toda la UNAH cada 15 minutos. 


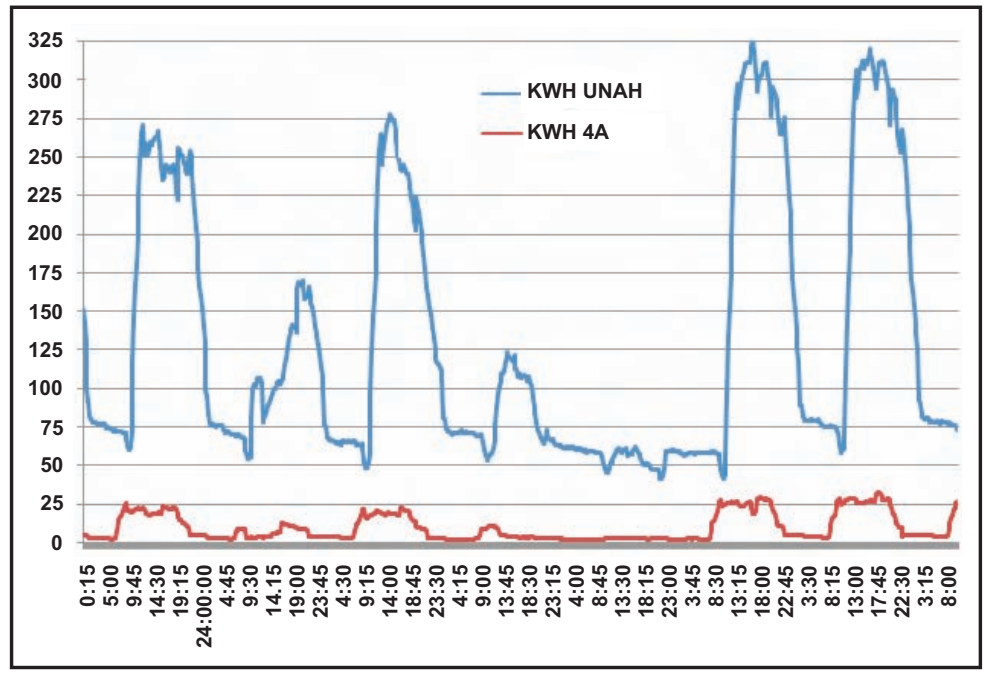

Fig. 7 Energía consumida cada 15 minutos en el edificio 4-A y toda la UNAH

En la figura anterior podemos ver que la gráfica de la UNAH con respecto a la del edificio 4-A, no presenta una similitud entre ambas, debido a que el consumo en el edificio 4-A durante ciertas horas es distinto con respecto a los edificios de toda la UNAH.

En la Fig. 8 se logra mostrar el gráfico de la energía acumulada tanto en el edificio 4A, como en toda la UNAH, a partir del 16 de abril hasta el 23 de abril del 2008 :

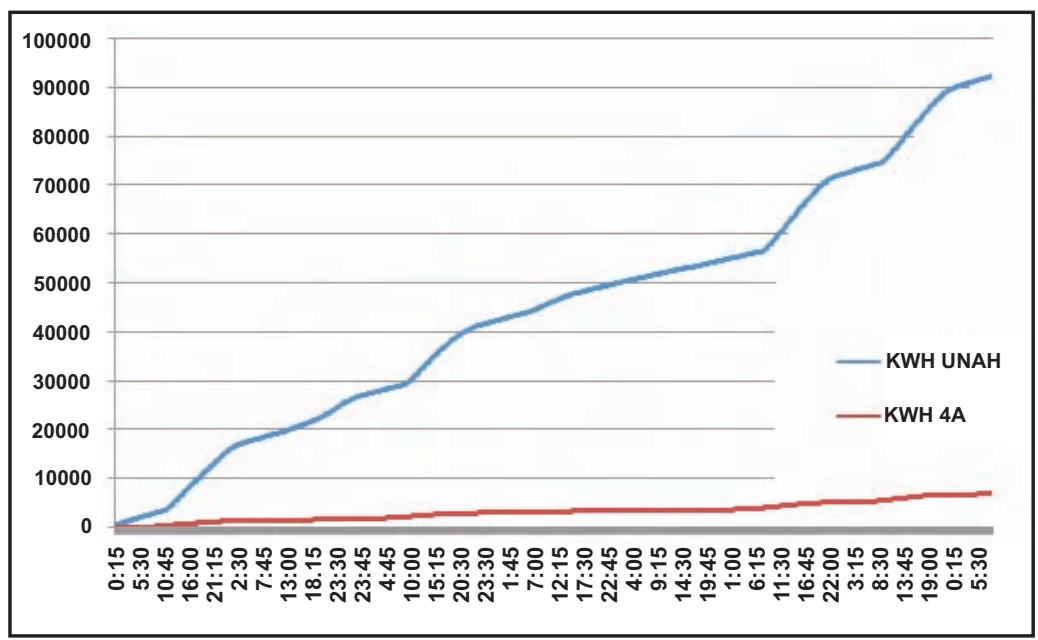

Fig. 8 Energia acumulada en el edificio 4-A y toda en la UNAH 
De la figura anterior, podemos concluir que el comportamiento de la gráfica de la energía acumulada es lineal, y así logramos obtener KWH total acumulados durante esta semana

Tabla III Tabla de KWH acumulados en el edificio 4-A y la UNAH

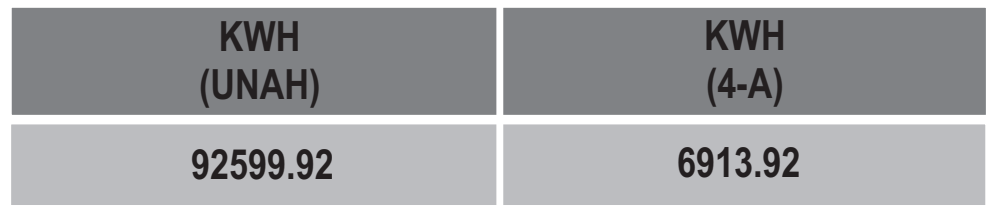

En la Fig. 9 logramos obtener un gráfico donde se muestra el porcentaje de la energía acumulada del edificio 4-A con respecto a la UNAH:

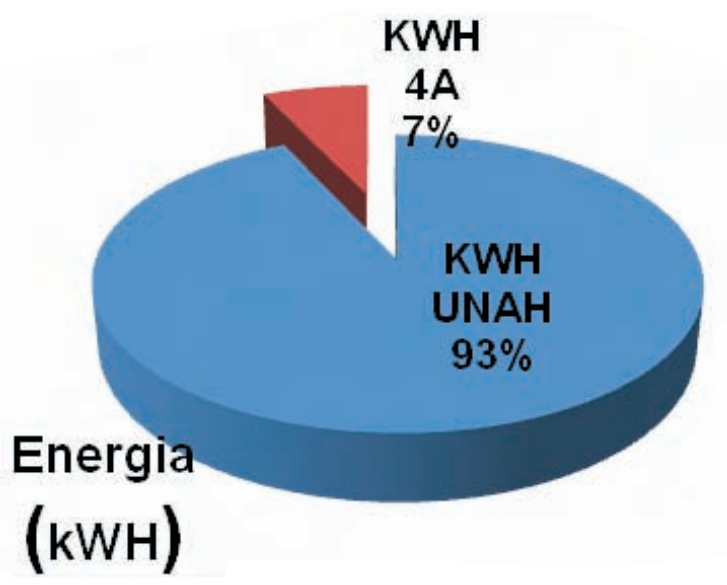

Fig. 9 Porcentaje de energía acumulada en el edificio 4-A y la UNAH

\section{Gráficos de corriente}

A partir de las mediciones que se obtuvieron en el analizador de red, logramos obtener la Fig. 10 en donde se muestran los diferentes gráficos de corriente que consume el edificio 4-A durante una semana, a partir del 16 de abril hasta el 23 de abril del 2008: 


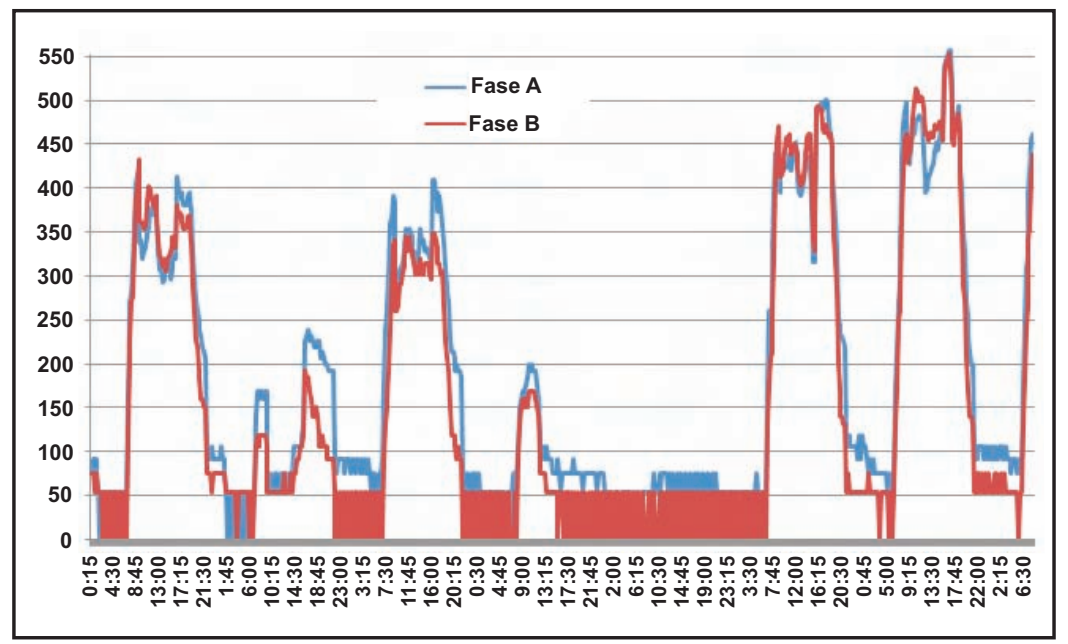

Fig. 10 Corrientes monofásicas en el edificio 4-A

\section{ANÁLISIS ECONÓMICO}

La demanda de potencia en el país ha crecido aproximadamente a 1,200 MW; se ha recurrido a implementar estrategias de ahorro de energía o a implementar el término de eficiencia energética, tanto en edificios públicos y privados, industrias y casas residenciales. En nuestro caso nos corresponde realizar este estudio económico de eficiencia energética aplicado al edificio 4-A de la UNAH, donde nuestro ahorro de energía se fundamenta en el uso adecuado del sistema de iluminación del edificio. Para lograr este propósito, es necesario realizar ajustes en la programación del temporizador, reduciendo las horas de control que tiene actualmente. También necesitamos del apoyo de la población estudiantil, de los maestros y de los empleados, para tener una conciencia de ahorro de energía y no derrochar en un gasto innecesario.

A continuación podemos ver los horarios actuales en los cuales se encuentra controlado el temporizador:

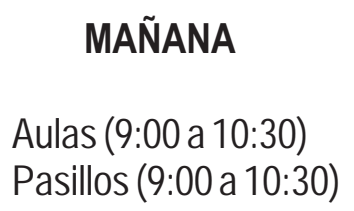

MAÑANA

Pasillos (9:00 a 10:30)

\section{TARDE-NOCHE}

Aulas (3:30 a 10:30)

Pasillos (3:30 a 10:30)

En la Fig. 11 podemos ver la conexión que tiene actualmente el temporizador que controla el edificio 4-A: 


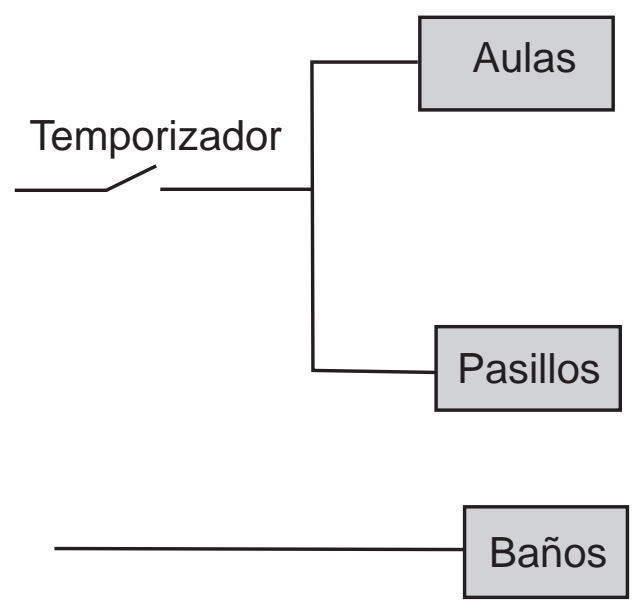

Fig. 11 Conexión del temporizador que tiene el edificio 4-A actualmente

Si hacemos un estudio del horario, nos damos cuenta que está distribuido de forma inadecuada, ya que la iluminación no es necesaria en ciertas horas del día; tampoco es necesaria en los pasillos por la mañana. También le agregamos que la iluminación de los baños permanece operando las 24 horas. En la Tabla IV mostramos la cantidad de horas en las que están funcionando actualmente, las lámparas de las aulas, de los pasillos y de los baños, así como el consumo diario y mensual de estas lámparas:

Tabla IV. Consumo actual en el sistema de iluminación del edificio 4-A

\begin{tabular}{|l|c|c|c|c|}
\hline & Potencia (KW) & $\begin{array}{c}\text { Horas de } \\
\text { funcionamiento }\end{array}$ & $\begin{array}{c}\text { Consumo } \\
\text { diario (KWH) }\end{array}$ & $\begin{array}{c}\text { Consumo } \\
\text { mensual (KWH) }\end{array}$ \\
\hline Baños & 2.88 & 24 & 69.12 & 2073.6 \\
\hline Pasillos & 4.68 & 8.5 & 39.78 & 835.38 \\
\hline Aulas & 9.4 & 8.5 & 79.9 & 1677.9 \\
\hline TOTAL & 37.96 & 61 & 398.8 & 4586.88 \\
\hline Lempiras & & & & $15,838.38$ \\
\hline
\end{tabular}

Partiendo de la información anterior, nuestra propuesta para lograr un ahorro de energía en el sistema de iluminación aplicado al edificio 4-A, consiste en modificar el horario de programación del temporizador; a la vez, hay que modificar el 
diagrama de conexión del temporizador. En la Fig. 12 podemos ver la nueva conexión que proponemos la cual consiste en que el temporizador controle los baños y pasillos y que las aulas queden independientes:
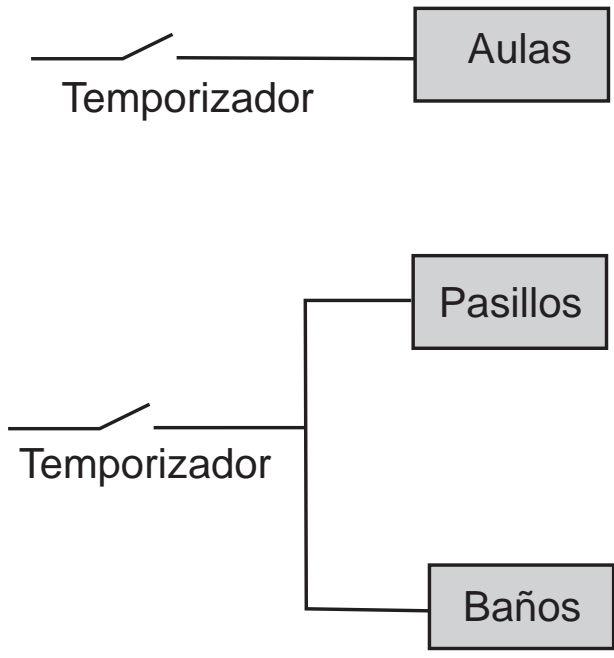

Fig. 12 Nueva propuesta de conexión aplicado al edificio 4-A

A continuación presentamos el nuevo horario propuesto, aplicado en el sistema de iluminación del edificio 4-A:

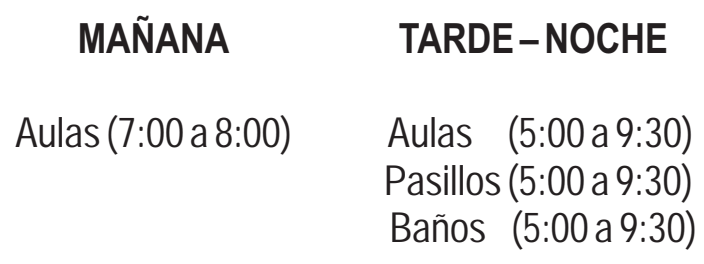

Con este nuevo horario logramos economizar energía y así reducir el pago de la factura a la ENEE; de igual forma alargamos el tiempo de vida de las lámparas. En la Tabla $\mathbf{V}$ se muestra la reducción de los tiempos en el funcionamiento de las lámparas con el cambio de conexión del temporizador y con la nueva programación del temporizador: 
Tabla V. Consumo referido a la nueva propuesta aplicada al sistema de iluminación del edificio 4-A

\begin{tabular}{|l|c|c|c|c|}
\hline Baños & Potencia (KW) & $\begin{array}{c}\text { Horas de } \\
\text { funcionamiento }\end{array}$ & $\begin{array}{c}\text { Consumo } \\
\text { diario (KWH) }\end{array}$ & $\begin{array}{c}\text { Consumo } \\
\text { mensual (KWH) }\end{array}$ \\
\hline Pasillos & 4.88 & 4.5 & 12.96 & 272.16 \\
\hline Aulas & 4.68 & 4.5 & 21.06 & 442.26 \\
\hline TOTAL & 16.96 & 5.5 & 51.07 & $1,085.70$ \\
\hline Lempiras & & 14.5 & 85.72 & $1,800.12$ \\
\hline
\end{tabular}

En base a los datos obtenidos en las Tablas IV y V, podemos comparar el ahorro que obtenemos al realizar la nueva propuesta de modificar la conexión del temporizador y programarlo correctamente. En la Tabla IV obtuvimos un consumo mensual de 4,586.88 KWH para la iluminación de las aulas, pasillos y baños, que traducido en el pago de la energía, corresponde a L. 15,838.38. En la Tabla V obtuvimos un consumo mensual de $1,800.12 \mathrm{KWH}$, es decir, que logramos un ahorro de 2,786.76 KWH. Estos 1,800.12 KWH corresponden a un pago de L. 7,537.64.

En la Tabla VI podemos ver el ahorro que obtenemos, tanto de $\mathrm{KWH}$, como en Lempiras:

Tabla VI. Tabla de ahorro

\begin{tabular}{|c|c|c|c|}
\hline $\begin{array}{c}\text { Lempiras } \\
\text { consumo } \\
\text { actual }\end{array}$ & $\begin{array}{c}\text { Lempiras } \\
\text { nuevo } \\
\text { consumo }\end{array}$ & $\begin{array}{c}\text { Lempiras } \\
\text { ahorrados } \\
\text { al mes }\end{array}$ & $\begin{array}{c}\text { Lempiras } \\
\text { ahorrados } \\
\text { al año }\end{array}$ \\
\hline $15,838.38$ & $7,537.64$ & $8,300.74$ & $83,007.4$ \\
\hline
\end{tabular}

\section{Ajuste del $120 \%$ por combustible.}

En la actualidad la ENEE cobra un ajuste por combustible de 120\%, debido a los incrementos que ha tenido el barril del petróleo. En la Tabla VII se muestra el nuevo consumo mensual de la UNAH: 
Tabla VII. Nuevo consumo mensual en la UNAH

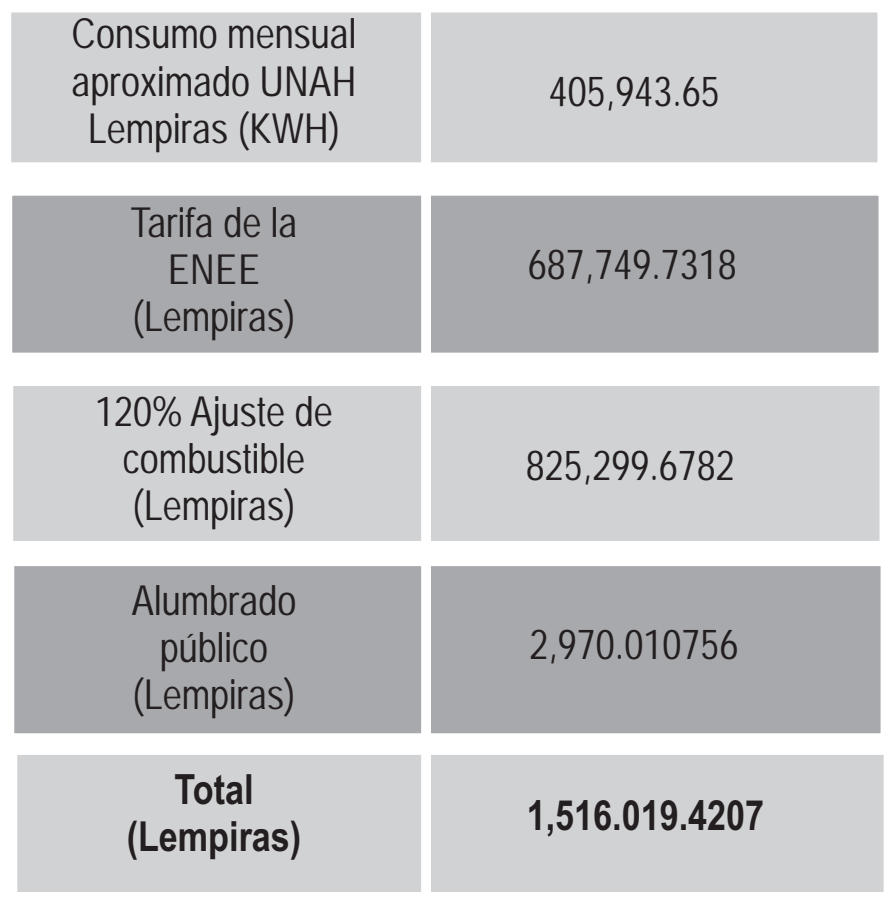

Tabla VIII. Nuevo consumo mensual en el edificio 4-A

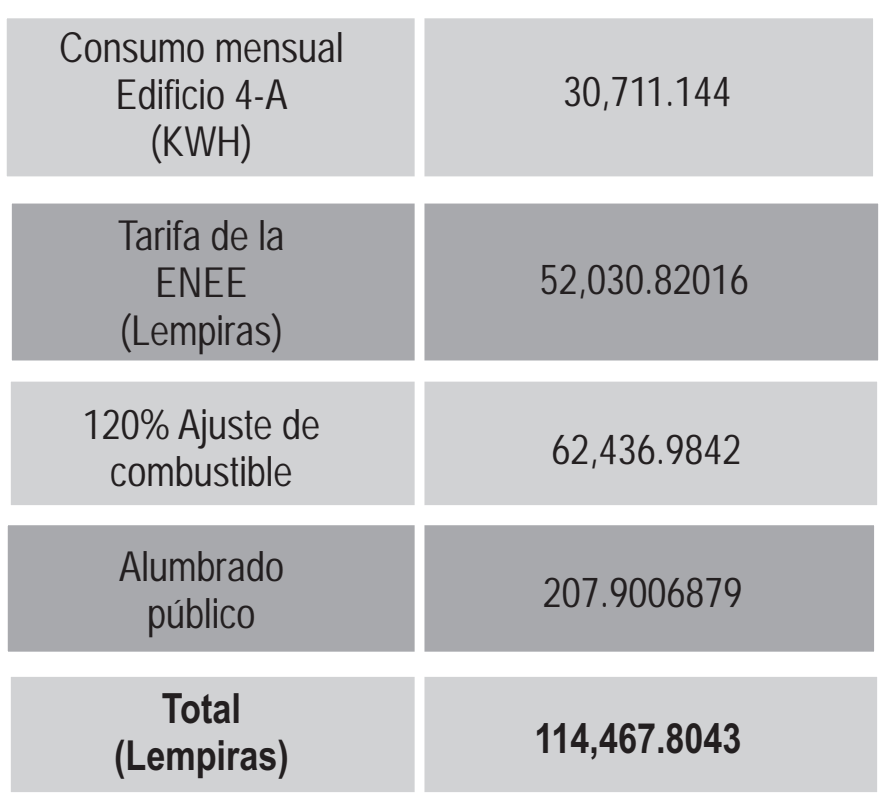

Dirección de Investigación Científica $\overline{121}$ 
Establecemos que con el nuevo ajuste de $120 \%$ al combustible, aumenta L. $309,487.38$ la factura mensual en la UNAH, mientras que en el edificio 4-A, la factura mensual tiene un aumento de L. 23,205.97.

\section{CONCLUSIONES}

Concluimos que a partir de la modificación de la conexión del temporizador y un ajuste adecuado en la programación de los tiempos, se logra un ahorro de energía de 2,786.76 KWH mensual, que trasladados al pago de la factura se ahorra una cantidad de L.8,300.74 mensuales. Si esto lo comparamos anualmente, tenemos que tomar como referencia que en la Universidad se trabaja aproximadamente 10 meses ( 2 meses aproximadamente son para vacaciones y feriados), entonces se logra un ahorro anual de L.83,007.40 en el pago de la factura de la ENEE.

\section{RECOMENDACIONES}

1) Modificar la conexión que tiene el temporizador que actualmente controla las lámparas de aulas y pasillos, y las lámparas de los baños se encuentran operando las 24 horas del día. A esto sugerimos que modifiquen la conexión del temporizador para que controle las lámparas de los baños y pasillos y las lámparas de las aulas queden independientes. También ajustar los tiempos de programación del temporizador, ya que actualmente se encuentra programado de una manera inadecuada.

2) Recomendamos cambiar con prontitud el transformador existente, por uno de mayor capacidad, ya que se registraron valores muy altos de corriente en el analizador de red. El transformador instalado es de 100 KVA con una corriente nominal de $417 \mathrm{~A}$, mientras que el analizador de red registró un valor máximo de corriente de $557 \mathrm{~A}$.

3) Motivar a través de rótulos publicitarios a la población estudiantil, al personal docente y administrativo, a realizar un uso racional de la energía eléctrica, y que estos rótulos se puedan colocar en los accesos principales de cada nivel, en las aulas, en los laboratorios y en los baños del edificio 4-A.

\section{AGRADECIMIENTO}

Agradecemos primeramente a Dios. Se agradece especialmente al compañero Gustavo Portales por toda su colaboración en la instalación de los equipos de 
medición; también se agradece el arduo trabajo de todos los alumnos que cursaron la asignatura de Seminario de Investigación en el tema de Eficiencia Energética, ya que por el trabajo de todos ellos se pudo realizar el levantamiento de carga de la UNAH.

\section{BIBLIOGRAFÍA}

Ayala L., Salinas F. y Andará, J. Proyecto de Eficiencia Energética aplicado a la Ciudad Universitaria. Seminario de Investigación (IE-900), III Periodo 2007.

"Mejora de la Eficiencia Energética en Instalaciones Industriales y Edificios". Disponible en:

http://www.icai.es/contenidos/publicaciones/anales_get.php?id=1443 